\title{
Primer registro fósil de un lapsino (Araneae, Salticidae) en el ámbar de Chiapas, México
}

\author{
Miguel Ángel García-Villafuerte
}

\begin{abstract}
Miguel Ángel García-Villafuerte
mgarciavillafuer@@yahoo.com.mx

Museo de Paleontología "Eliseo Palacios Aguilera". Dirección de Paleontología. Secretaría de Medio Ambiente e Historia Natural. Calzada de los hombres Ilustres s/n. G.P. 29000. Antiguo Parque Madero. Tuxtla Gutiérrez, Chiapas; México.
\end{abstract}

BOL. SOC. GEOL. MEX. 2018

VOL. 70 NO. 3

P. $689-708$

http://dx.doi.org/10.18268/BSGM2018v70n3a6

\section{RESUMEN}

Se reporta el primer lapsino (Salticidae: Spartaeinae) fosilizado en ámbar de Chiapas, México. Este espécimen es una nueva especie del actual género Galianora, con una edad de 23 Ma (MiocenoAquitaniano). Este es el primer lapsino en el registro fósil, el grupo aún no se ha registrado en la actual araneofuna chiapaneca. Este registro extiende el rango geográfico del género hacia el norte de la región Neotropical, con un representante fósil. Esta nueva especie, y los registros previos de salticidos para el ámbar mexicano, son consistentes con una radiación de Salticidae en el Neógeno, con implicaciones tanto Neárticas como Neotropicales. La presencia del género Galianora en el ámbar, predice su existencia en la actual fauna de Chiapas. Con base en el registro fósil se discute la idea de que probablemente los géneros de arañas, aparentemente extintos, aún permanecen en la región y posiblemente sobrevivieron a la evolución geológica de América Central. Se podría considerar que Chiapas fue, en su momento, un centro de origen para varias especies del órden Araneae; por lo cual, es necesario considerar la intensificación de estudios araneofaunísticos enfocados en analizar la riqueza y diversidad de arañas en Chiapas, considerando estudios filogenéticos (que incluyan taxones fósiles) para probar esta hipótesis.

Palabras clave: palabras, clave, de, muestra. araña saltadora, Galianora, Simojovel, fósil.

\section{ABSTRACT}

The first lapsine jumping spider (Salticidae: Spartaeinae) is recorded from Chiapas amber, Mexico. This specimen is a new fossil species belonging to the extant genus Galianora, with an age of 23 My (Miocene-Aquitanian). This is the first lapsine in the the fossil record, a group not previously recorded in the extant spider fauna from Chiapas. The geographical range of the genus extends towards the north of the Neotropical region thanks to this fossil representative. This new species, and previous records of salticids in Mexican amber, are consistent with a radiation of Salticidae in the Neogene, with both Neartic and Neotropical implications. The presence of Galianora in amber, predicts its presence in the extant Chiapas spider fauna. Based on the fossil record, it is possible that extinct genera of spiders may still remain in the region and probably survived the geological evolution of Central America. It is also possible that Chiapas was a center of origin for several species of Araneae; therefore, it is necessary to intensify araneofaunistic studies focused on analyzing the richness and diversity of spiders in Chiapas, using phylogenetic studies (including fossil taxa) to test this hypothesis.

Keywords: jumping spider, Galianora, Simojovel, fossil. 


\section{Introducción}

El ámbar mexicano, localizado en el municipio de Simojovel de Allende, Chiapas; fue producido por una especie de árbol de la familia Fabaceae, Hymenea mexicana (Poinar y Brown, 2002, citado en Bickel y Solórzano-Kraemer, 2016). La resina fosilizada en esta región ha sido datado con una edad geológica de $23 \mathrm{Ma}$, correspondiente al Mioceno Inferior (Aquitaniano) (Vega et al., 2009; Perrillat et al., 2010; Serrano-Sánchez et al., 2015). Simojovel constituye uno de los depósitos ambarinos más importantes del mundo y en él se han encontrado alrededor de 200 familias de artrópodos (Solórzano-Kraemer, 2010). Muchas de las investigaciones realizadas con inclusiones biológicas en ámbar de esta región, han sido centradas en los diferentes Órdenes de la Clase Insecta (e.g. Macadam y Ross, 2016; Popov, 2016; Ross et al., 2016a, 2016b; Heiss, 2016); otros más con crustáceos (e.g. Serrano-Sánchez et al., 2016), plantas (e.g. Hernández-Damián et al., 2016) y arácnidos (e.g. Rivas et al., 2016; Judson, 2016), entre estos últimos las arañas (Petrunkevitch, 1963, 1971; GarcíaVillafuerte y Penney, 2003; García-Villafuerte 2004, 2006a, 2006b, 2007, 2008). Actualmente, el órden Araneae se compone de 112 familias, la más numerosa de ellas es Salticidae (World Spider Catalog, 2018).

Los miembros de la familia Salticidae están presentes en todos los continentes con excepción de la Antártida, dentro de una amplia gama de micro hábitats, desde la hojarasca hasta la copa de los árboles (Richman et al., 2005). Dado su amplio rango de distribución es la familia con mayor riqueza de especies dentro del órden Araneae, representando el $10.68 \%$ del total de especies de arañas válidas registradas para el mundo (World Spider Catalog, 2018). A nivel mundial, se han registrado un total de 6077 especies actuales, distribuidas en 634 géneros (World Spider Catalog, 2018). El conocimiento de la riqueza de la fauna de arañas saltadoras para México se conoce a través de los trabajos realizados por Hoffman (1976), Jiménez (1996), Castelo-Calvillo (2000) y Richman et al. (2011); a la fecha se han reportado 264 especies distribuidas en 65 géneros para el país (Richman et al., 2011). En lo que respecta al estado de Chiapas se han registrado 25 especies válidas, incluidas en 14 géneros (Richman et al., 2011; Garcilazo-Cruz y Álvarez-Padilla, 2015).

En lo que concierne al registro fósil de Salticidae, se enlistan las especies en Dunlop et al. (2018), las cuales han sido reportadas para los diferentes depósitos ambarinos; destacando principalmente el Báltico y la República Dominicana. Para el caso del ámbar mexicano los registros son escasos, incertae sedis (Petrunkevitch, 1971), Lyssomanes sp. (García-Villafuerte y Penney, 2003), Maevia? eureka Riquelme y Menéndez-Acuña, 2017, gen. sp., indet. (García-Villafuerte, 2004) y cinco gens. sp., indeterminados, que se encuentran resguardados en museos extranjeros (Ross et al., 2016b).

En el presente estudio se reporta una nueva especie fósil del actual género Galianora Maddison, 2006, de la tribu Lapsiini perteneciente a la subfamilia Spartaeinae, encontrada en ámbar de Chiapas, México. Hill y Richman (2009) mencionan que aunque muchos grupos de salticidos basales se encuentran en los trópicos del Viejo Mundo y en las Indias Orientales en particular; otros, incluyendo muchos lyssomaninos y lapsinos actuales, están ampliamente distribuidos en los trópicos del Nuevo Mundo.

La presencia de un lapsino en el ámbar de Chiapas, permite reflexionar puntos importantes, como detalles taxonómicos de la familia, aspectos biogeográficos del género y su posible permanencia en la fauna actual, también se discute el tema del origen de la familia Salticidae y su divergencia; finalmente, con base en el registro fósil y actual de arañas, se plantea la idea de que los géneros araneidos, aparentemente extintos, pueden permanecer en la región, y su probable sobrevivencia a la evolución geológica de América Central. 


\section{2. Área de Estudio y Método}

La pieza de ámbar estudiada proviene del municipio de Simojovel de Allende en el estado de Chiapas, que está ubicado a $50 \mathrm{~km}$ de la ciudad de Tuxtla Gutiérrez, al norte del estado y al sur de México (Figura 1). En el área, la resina fósil es extraída a través de túneles de depósitos marinos. Las minas principales son los Pocitos, Campo La Granja y El Pistón, donde interactúan tres unidades litoestratigráficas: Formación La Quinta, Mazantic y Balumtum (Figura 2); éstas unidades están expuestas en la Sierra Madre del Sur de Chiapas, desde el borde de la Depresión Central (Totolapa) hasta Palenque, cerca de las costas del Golfo de Tabasco (Alisson, 1967; Frost y Langenhein, 1974; Perrillat et al., 2010; Riquelme et al., 2014; Serrano-Sánchez et al., 2015).

El espécimen fósil en ámbar proviene de la mina los Pocitos, fue adquirida directamente con un artesano de la localidad por el Biólogo Marco Antonio Coutiño José, quien la donó al Museo de Paleontología "Eliseo Palacios Aguilera", Dirección de Paleontología de la Secretaria de Medio Ambiente e Historia Natural (SEMAHN), donde actualmente se resguarda bajo el número de catálogo: IHNFG-5494 (Instituto de Historia Natural, Fósil Geográfico).

Para obtener una mejor visualización del espécimen, se utilizaron lijas de diferentes medidas, con

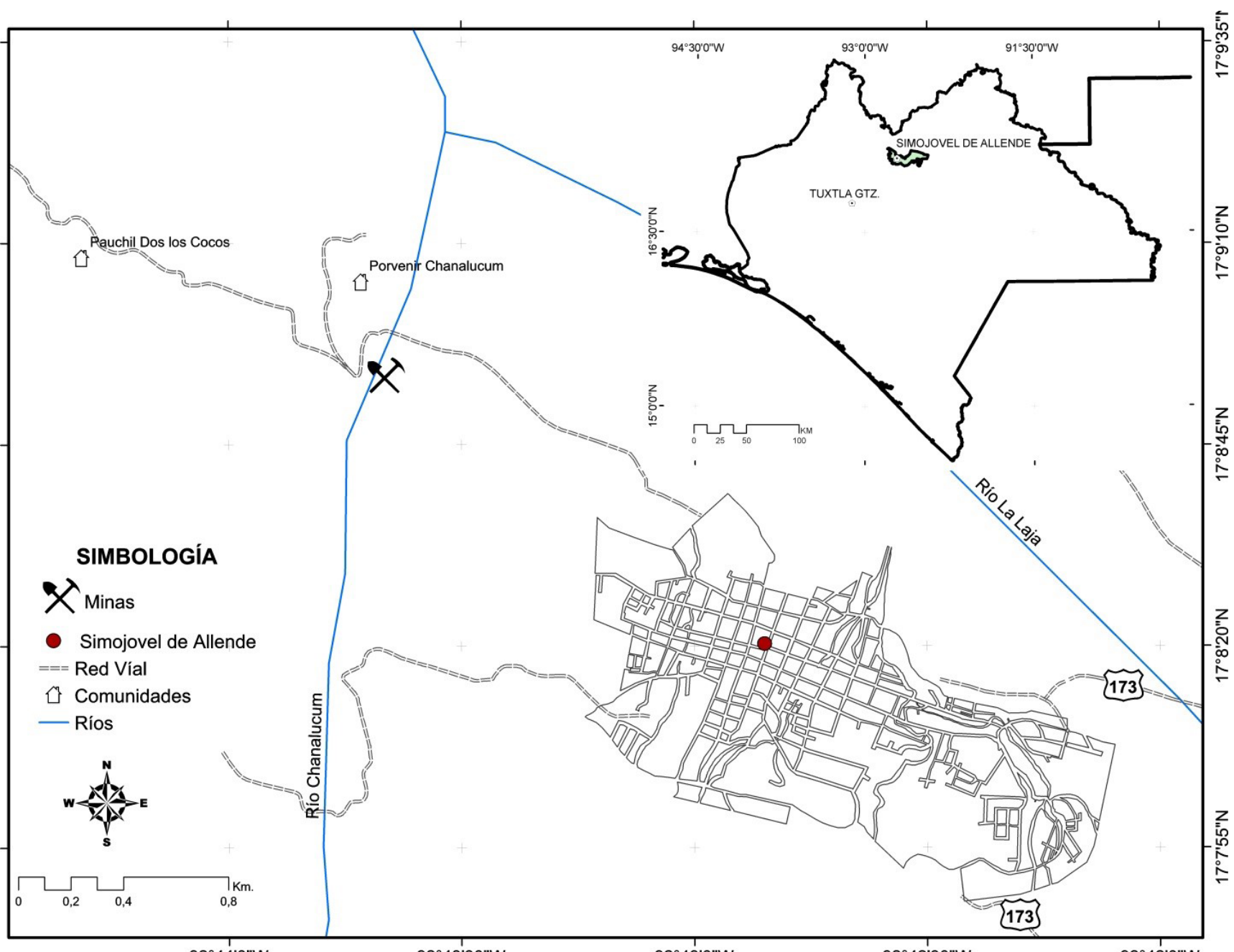

$92^{\circ} 44^{\prime} 0^{\prime \prime} \mathrm{W}$

$92^{\circ} 43^{\prime} 30^{\prime \prime} \mathrm{W}$

$92^{\circ} 43^{\prime} 0^{\prime \prime} \mathrm{W}$

$92^{\circ} 42^{\prime} 30^{\prime \prime} \mathrm{W}$

$92^{\circ} 42^{\prime} 0^{\prime \prime} \mathrm{W}$

Ubicación de la mina "Los Pocitos" en Simojovel, Chiapas; México. 

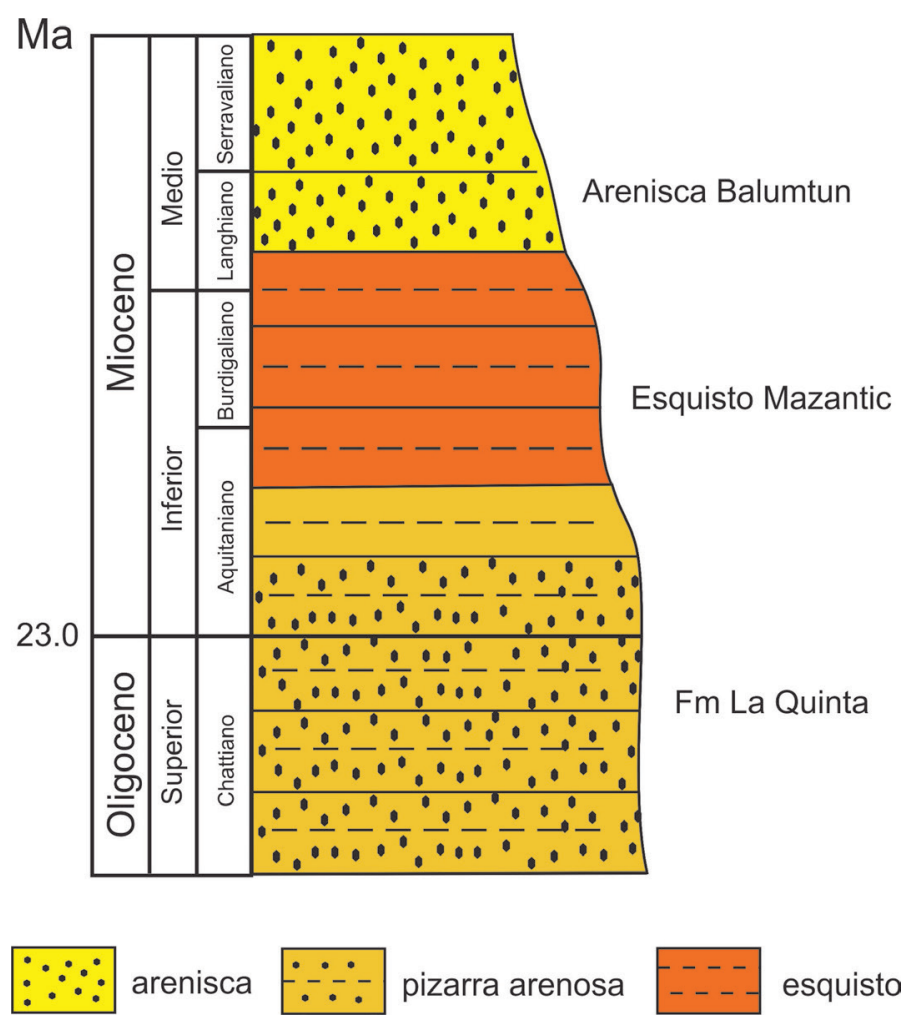

Figura 2 Estratigrafía general del área de estudio, modificado de Serrano-Sánchez et al. (2015).

el fin de aminorar el tamaño de la pieza y finalmente, se utilizó un abrasivo de la marca Brasso ${ }^{\circledR}$, para pulir la pieza, que entre sus múltiples usos está el eliminar y reparar arañazos en los plásticos, CD's, DVDS, pantallas y piscinas; el uso de este material en el ámbar ha demostrado que elimina las ralladuras que deja la lija más fina. Las fotografías fueron realizadas con una cámara Leica MC170HD conectada a un microscopio estereoscópico Leica M205C. Utilizando la técnica de apilamiento se realizaron una serie de tomas de las estructuras bajando el enfoque gradualmente; posteriormente, con el software Helicon focus 6, las fotografías se sobrepusieron para lograr una composición con amplia profundidad de campo y enfoque; las imágenes se importaron a Ligth room 5.5 para el proceso de revelado. La descripción del espécimen se da con base en lo propuesto por Galiano (1963) y Maddison (2006). Las medidas son dadas en milímetros y fueron tomadas con una gratícula ocular adaptada a un Estereoscopio Zeiss Stemi 2000. Abreviaturas: AO (área ocular),
OMA (ojos medios anteriores), OLA (ojos laterales anteriores), OMP (ojos medios posteriores), OLP (ojos laterales posteriores), Gi (cimbio), E (émbolo), Am (apófisis media), Te (tegulum), Bc (pestaña del cimbio), fe (fémur), pat (patela), tib (tibia), mt (metatarso), es (espina), r (retrolateral), p (prolateral), pa (prolateral apical).

\section{Paleontología sistemática}

Órden Araneae Clerck, 1757

Familia Salticidae Blackwall, 1841

Genero Galianora Maddison, 2006

Especie tipo: Galianora sacha Maddison, 2006

Galianora marcoi sp. nov. (Figuras 3a-f).

Material examinado. Holotipo macho IHNFG5494 en ámbar de Simojovel de Allende, Chiapas México. Mioceno (Aquitaniano) - 23 Ma. 
Diagnosis. Galianora marcoi sp. nov., se diferencia de las dos especies actualmente descritas, Galianora bryicola Maddison, 2006 y G. sacha Maddison, 2006, por la forma de la apófisis medía que es alargada, recta, en forma de muleta; la punta del émbolo es más grueso, semibifurcado, se erige distalmente en el lado retrolateral y por la presencia de una pestaña retrolateral en la base del cimbio en forma de cuernos.
Etimología. El epíteto específico es un nombre en aposición y es dedicado a Marco Antonio Coutiño José (Museo de Paleontología "Eliseo Palacios Aguilera"), por la importante labor en la adquisición de piezas significativas de ámbar con inclusiones biológicas, las cuales ha donado al Museo de Paleontología antes mencionado, y por su interés en el conocimiento de la araneofauna fósil chiapaneca.


Figura 3 Galianora marcoi sp. nov., a: vista dorsal; b: vista ventral; c: vista lateral, escala: 1 mm; d: Palpo, vista prolateral; e: Palpo, vista dorsal; f: dibujo de Palpo, vista prolateral, escala: $0.3 \mathrm{~mm}$. 
Descripción del macho holotipo (Figuras 3a-f). Longitud total 3.72. Longitud prósoma 1.76, ancho 0.98, alto: 0.58; AO: largo 0.84, línea anterior de ojos: 1.03 ancho, línea posterior ojos 0.78; estría torácica alargada. Distancia entre OLA y OMP 0.14; distancia entre OMP y OLP 0.21; altura del clípeo: es difícil tomar la altura por la posición de la araña, pero se observa un clípeo alto. Diámetro de OMA 0.29: Patas IV, I, III, II aproximadamente. Quetotaxia: I fe1-1 d; pat 0; tib 1-2-2 v, 1pt; mt 2-2 v. II fe 1-1d; pat 0 ; tib 1-1-1 r; mt 2-2v, con un verticilio apical de 4 es. III fe 1-1-1d, 1pa, pat 1p; mt 1v, 1-1r, 1p, con un verticilio apical de 4 es. IV fe 1-1d, pat 1r; tib 1-1r, 1-1p; mt 1-1-1p. Palpo: fe 0.58, pat?, tib 0.31 , ci 0.54 ; el cimbio tiene una forma cónica, la punta se observa esclerozada con un patrón de setas medianas agrupadas de tal manera que tienen la apariencia de un estilo punk en una vista lateral (Figura 3b). En vista dorsal se observa una pestaña en la base retrolateral del cimbio, que tiene la forma de dos cuernos uno más grande que otro (Figura 3e). Prolateralmente se logra observar un tegulum redondeado, el émbolo periférico distintivo del género y la apófisis media delgada y alargada, recta, en forma de muleta, 0.089 de longitud (Figuras $3 \mathrm{~d}$ y f), dorsalmente se observa el extremo distal del émbolo de manera ensanchada y semibifurcada (Figura 3e). La ART tiene forma de un cáliz y está fuertemente esclerosado. Opistosoma: 1.69 de longitud.

Aspecto y color en ámbar. el prósoma no es muy elevado y presenta un suave declive torácico, con una fóvea larga. En el centro hay una banda ancha, con setas amarillentas y blanquecinas, que nace muy cerca de la base de los OMP y desemboca cerca del pedicelo terminando en punta; ambos costados de la base del prosoma de color negro y de la parte media hacia la línea de ojos anteriores de color pardusco. En la base de los OLP se observan sedas de color anaranjado oscuro, el mismo color se presenta en la base de los OMP hacia los OMA; los quelíceros son de color café claro; el opistosoma es de color pardo amarillento y todas las patas de color amarillo con ciertos tonos moteados pardos.

Hembra: desconocida.

\section{Discusión}

\subsection{DETALLES TAXONÓMICIOS}

El espécimen estudiado ha sido ubicado dentro de la familia Salticidae, ya que las arañas saltadoras se caracterizan por la presencia de grandes ojos medios anteriores (OMA) (Scheuring, 1914; Ramírez, 2014) y por el característico arreglo de los ojos (Maddison, 2015). Penney (2010) menciona que esta característica es muy distintiva en los salticidos por lo que pueden identificarse incluso a partir de especímenes juveniles, aun cuando se encuentran en la resina fosilizada. Coddintong y Levi (1991) señalan que la sinapomorfia más sobresaliente de la familia es el par de ojos medios anteriores altamente especializados.

Hill y Richman (2009) mencionan que el desarrollo único de la visión en Salticidae ha respaldado la evolución de una gama extraordinariamente diversa de estilos de vida, todos asociados con un comportamiento visualmente mediado. Los salticidos son miembros del clado ART (por la apófisis retrolateral en los machos), este clado incluye por lo menos la mitad de las arañas conocidas (Blackledge et al., 2009) y con pocas excepciones, la familia está entre los miembros del clado que aparentemente perdieron la capacidad de construir telarañas (Hill y Richman, 2009).

Determinar si una araña pertenece o no a la familia Salticidae es relativamente sencillo, pero la ubicación taxonómica de un espécimen dentro de una subfamilia, género o especie en particular, requiere una detallada observación, no solo de los caracteres somáticos, sino también de las estructuras genitales, sean machos o hembras. Harms y Dunlop (2008) mencionan que las arañas fósiles en ámbar a menudo se asemejan incluso a géneros y especies actuales y por lo general se pueden ubicar dentro de familias actuales. Pero también son difíciles de comparar con otros fósiles o especies 
cercanas, debido a que una observación clara de los genitales (palpo, en los machos y/o epiginio, en las hembras) a menudo es difícil de distinguir. En efecto, los paleoaracnólogos se enfrentan a una serie de dificultades al estudiar arañas fósiles incluidas en ámbar; por ejemplo, la posición en la cual quedan fosilizados los especímenes, las fracturas y burbujas que obstruyen la visibilidad de estructuras clave en la determinación taxonómica, etc. En este caso, los palpos del espécimen estudiado se encuentran doblados hacia dentro, de tal manera que el dorso del cimbio se observa ventralmente (Figura 3b). Al principio de la investigación esto dificultó su determinación taxonómica; consecuentemente, se tuvo que lijar y pulir la pieza por la parte posterior, del lado del opistosoma, teniendo el cuidado de no dañarla a fin de observar las estructuras genitales. $\mathrm{Al}$ hacer esto, se logró visualizar uno de los palpos en una vista prolateral, observando así el tegulum, el émbolo y la apófisis media (Figuras 3d y 3f). Harms y Dunlop (2008) mencionan que los caracteres del pedipalpo masculino en las arañas, representan la característica taxonómica más importante para su determinación. De la misma manera, Prószyński (2017) menciona que los palpos son estructuras conservadoras, estables, que caracterizan amplios 'grupos de géneros', con pequeñas modificaciones en los detalles en relación con géneros y especies. Estas son las únicas estructuras que permiten, apenas dándoles un vistazo, la identificación de subfamilia o géneros.

A continuación se argumenta porqué el espécimen fósil estudiado se ha ubicado dentro del actual género Galianora. Después de comparar el ejemplar con claves taxonómicas de arañas actuales para el norte de América (Richman et al., 2005), consultar diferentes bibliotecas ilustradas sobre salticidos (e.g. Prószyński, 2015; Metzner, 2015) y visitar el catalogo mundial de arañas (World Spider Catalog, 2018); se consideró el trabajo de Maddison (2015), quien realiza una clasificación filogenética de las arañas saltadoras con base en análisis morfológicos y moleculares, un trabajo valioso para el estudio de la evolución de salticidos (Prószyński,
2017). Maddison (2015) establece siete subfamilias: Onomastinae, Asemoneinae, Lyssomaninae, Spartaeinae, Eupoinae, Hisponinae y la más numerosa de ellas, Salticinae.

Con base en la descripción de cada una de las subfamilias, Galianora marcoi sp. nov., fue excluida de Onomastinae, Asemoneinae y Lysomaninae ya que carece de la translucencia verde o amarilla que identifica a las antes mencionadas, así como el característico patrón de ojos que diferencia a sus miembros. También fue descartada de la Subfamilia Eupoinae porque no tiene un cuerpo pequeño brillante y una escuta en el dorso del abdomen. Se separó de la subfamilia Hisponinae debido a que no presenta el surco o constricción transversal en el caparazón, justo detrás de los ojos medios posteriores (ver figura 23 en Maddison, 2015). También fue diferenciada de la subfamilia Salticinae porque el palpo de Galianora marcoi sp. nov., presenta una apófisis media; por lo tanto, se ubicó como miembro de la Subfamilia Spartaeinae.

Maddison (2015) menciona que las especies que componen a Spartaeinae, no tienen sinapomorfias morfológicas conocidas y aparentemente están unidos por estados de carácter posiblemente ancestrales; sin embargo, señala que los subgrupos o tribus que la componen, se encuentran claramente definidos por un rango geográfico, más que por su morfología. La subfamilia Spartaeinae se compone de tres tribus, una de ellas es Sparteini, principalmente de distribución africana, asiática, con pocos representantes en Europa y Australasia; la Tribu Cocalodini, cuyas especies (a excepción de Depreissia) están restringidas a Australasia, al este de la línea de Wallace (Wanless, 1982; Maddison, 2006) y son comunes en la araneofauna de Nueva Guinea, con variadas formas corporales (Maddison, 2006 citado en Maddison, 2015). Galianora marcoi sp. nov., fue descartado de estas dos tribus debido a que las investigaciones hasta hoy realizadas (García-Villafuerte y Penney, 2003; García-Villafuerte, 2006a, 2006b, 2008) indican que la araneofauna fósil en el ámbar de Chiapas es similar a la reciente fauna Neotropical. Además, 
se revisaron los artículos relacionados a los géneros de cada tribu, que presentan una apófisis media en el palpo, para comparar la estructura genital con el espécimen estudiado, y existen diferencias significativas (e.g. Wanles, 1982, 1984; DeelemanReinhold y Floren, 2003; Logunov y Azarkina, 2008; Prószyński y Deeleman-Reinhold, 2012; Maddison et al., 2014; Prószyński, 2017 y otros en: World Spider Catalog, 2018).

La tercer tribu, llamada Lapsiini, tiene una distribución Neotropical en el Nuevo Mundo y aunque no hay sinapomorfías que distingan a este grupo (Maddison, 2015), las especies que la integran, al igual que los lyssomaninos, comparten un rasgo ancestral adicional, la presencia de una apófisis media en el palpo del macho y la uña en los palpos de las hembras, lo cual no se observa en otras especies de salticidos neotropicales (Maddison 2006). Por otro lado, se revisó la descripción de las especies fósiles de arañas saltadoras con apófisis media en el palpo, según descritas en Wunderlich (2004a), que han sido registradas tanto para el Báltico como para la República Dominicana y Galianora marcoi sp. nov., muestra marcadas diferencias en cuanto a la posición de la apófisis media y la forma del émbolo y el tegulum. Por lo tanto, se llegó a la conclusión que el espécimen estudiado forma parte de la subfamilia Spartaeinae, como miembro de la tribu Lapsiini, siguiendo lo establecido por Maddison (2015).

La tribu Lapsiini, contiene 20 especies válidas distribuidas en cinco géneros actuales, Galianora Maddison, 2006; Lapsamita Ruiz, 2013; Lapsias Simon, 1900; Soesiladeepakius Makhan, 2007 y Thrandina Maddison, 2006 (Maddison, 2015; Prószyński, 2017; World Spider Catalog, 2018).

De los géneros que componen a los lapsinos, Galianora marcoi sp. nov., se corrobora con la descripción del género Galianora, ya que se diagnostica entre los salticidos neotropicales por poseer un tegulum redondeado con un émbolo periférico (Figura 3d y 3f). Las hembras son reconocidas por la presencia de una uña en los palpos. Se llegó a la misma conclusión, según lo establecido por Prószyński (2017), sin embargo, él menciona que las hembras se caracterizan por tener un epiginio con espermatecas y ductos muy elaborados o complicados.

Para este género se han descrito dos especies: Galianora bryicola Maddison, 2006 y G. sacha Maddison, 2006. Galianora marcoi sp. nov., tiene un gran parecido, en la forma del cuerpo, con $G$. bryicola, pero se diferencia de esta por la forma del émbolo, la apófisis media y la ART. Por otro lado, el émbolo es muy parecido a $G$. sacha; sin embargo, Galianora marcoi sp. nov., se diferencia de ella por la forma de la apófisis media, la cual es más larga y delgada, en forma de muleta y no tiene el cuerpo alongado, sino más bien compacto. Y principalmente, por la presencia de una pestaña retrolateral en la base del cimbio en forma de cuerno (Figura 3e), se establece Galianora marcoi sp. nov. Al parecer, Galianora marcoi sp. nov., es una especie intermedia en la forma del cuerpo y el palpo.

\subsection{ASPECTOS BIOGEOGRÁFICIOS}

Después de la descripción del género tipo, Lapsias Simon (1900), ninguna otra especie fue correctamente agregada a la tribu Lapsiini por más de un siglo (Maddison, 2006). De acuerdo con Maddison (2015), los lapsinos neotropicales están confinados al Nuevo Mundo; miembros del género Lapsamita han sido reportados para Brasil, el género Lapsias para Ecuador y Venezuela, Soesiladeepakius para Suriname y Brasil, Thrandina tiene presencia en Ecuador (Maddison, 2006).

Hasta hoy día, no se había reportado al género Galianora en el registro fósil, y tampoco se tiene contemplada para el catálogo de especies actuales en el Estado de Chiapas (García-Villafuerte, 2009; Ibarra-Núñez, 2013) o en el listado a nivel Nacional en México (World Spider Catalog, 2018). El único estudio a la fecha sobre las especies que lo componen, las ubica geográficamente en Ecuador (Maddison, 2006; World Spider Catalog, 2018) y algunas especies aún no descritas para Venezuela (Maddison, 2006). La presencia de Galianora marcoi sp. nov., en el ámbar de Chiapas, proporciona información paleobiogeográfica importante sobre las actuales especies sudamericanas, ampliando el 
rango de distribución del género hacia el norte de la región Neotropical. Un caso similar ocurre con Maevia? eureka, una especie de araña de la subtribu Marpissina, encontrada en el ámbar de Totolapa, cerca de Simojovel; la cual extiende el rango geográfico del género, actualmente restringido a Norteamérica, hacia una región meridional. Tampoco este género ha sido reportado en el listado de especies actuales del órden Araneae para Chiapas (García-Villafuerte, 2009; Richman et al., 2011; Ibarra-Núñez, 2013).

\section{3. ¿GÉNEROS EXTINTOS?}

Riquelme y Menéndez-Acuña (2017) sugieren, por la presencia de Maevia? eureka en el ámbar de Totolapa (Chiapas), y que coincide con otro registro actual al norte de México (Tamaulipas), M. poultoni Peckham y Peckham, 1901 (Richman et al., 2011), que muy probablemente el género Maevia? se dispersó a lo largo de los neotrópicos en el Mioceno y que finalmente se extinguió en el sur, manteniéndose casi exclusivamente en la región Neártica.

Esta sugerencia es sin duda precipitada; en primer lugar, Penney et al. (2003) mencionan el caso de la familia Archaeidae, la cual fue descripta por primera vez a partir de registros fósiles en el ámbar del Báltico por Koch y Berendt (1854); posteriormente en 1881, O.P. Cambridge descubre un archaeido vivo en Madagascar, Eriauchenius workmani O. Pickard-Cambridge, 1881 (Wood, 2008). Más de un siglo después se han registrado nuevas especies actuales para esta familia, que se consideraba extinta, en Madagascar, Sudáfrica y Australia (World Spider Catalog, 2018). En segundo lugar, debido a la complejidad y diversidad de ambientes, el estudio de la araneofauna actual en Chiapas es aún insuficiente y hasta la fecha no se tienen registros válidamente reportados de arañas para los municipios chiapanecos de Simojovel y Totolapa; y aún queda mucho territorio por muestrear en el Estado (ver mapa 1 en García-Villafuerte, 2009 y el último listado araneofaunístico en Ibarra-Núñez, 2013). En tercer lugar, sobreviene el caso del género Hemirrhagus Simon, 1903, una tarántula de la familia Theraphosidae, a quien en un principio se registró como representante de la araneofauna fósil chiapaneca (García-Villafuerte, 2008) y posteriormente, como parte del proyecto "Fortalecimiento de la Colección de Arácnidos de la Universidad de Ciencias y Artes de Chiapas", se reportó la presencia del género dentro del listado de arañas actuales para Chiapas (García-Villafuerte, 2009), y que resultó ser una nueva especie para la ciencia, Hemirrhagus perezmilesi García-Villafuerte y Locht, 2010 ¿Podría ocurrir el mismo caso con Maevia?, Galianora y los géneros aparentemente extintos reportados por Petrunkevitch (1963, 1971)? ¿Es posible que hoy día haya representantes actuales de estos géneros en Chiapas?

Por lo tanto, podríamos esperar que representantes de los géneros Maevia? y Galianora, reportados como registros fósiles en el Estado de Chiapas, se encuentren actualmente en esta región del mundo, como sucede con los géneros Hemirrhagus, Episinus, Selenops y Lyssomanes (García-Villafuerte y Penney, 2003; García-Villafuerte, 2006a，2006b，2008, 2009; García-Villafuerte y Locht, 2010), los cuales tienen representantes en la actual araneofauna chiapaneca, aun después de 23 Ma. Pero para constatar esto, es importante realizar investigaciones sobre la riqueza de arañas actuales aledañas a las minas de Simojovel y otros municipios; probablemente estos géneros aun ocupan su hábitat, manteniéndose en el tiempo desde el Mioceno (Aquitaniano) a la fecha.

\subsection{ORIGEN Y DIVERGENGIA DE SALTICIDAE}

El origen de la familia Salticidae fue tema de debate; se pensaba que la familia se había originado en el Mesozoico (durante el Cretácico), con base en los registros publicados por Grimaldi et al. (2002), quienes registraron una araña saltadora para el ámbar de Nueva Jersey; al igual que Néraudeau et al. (2002), al publicar la existencia de Salticidae en el ámbar de Francia y por un espécimen reportado como salticido por Kaddumi (2005) para el ámbar de Jordania (Penney et al., 
2003); sin embargo, Penney (2007) menciona que tales determinaciones son erróneas y dichos organismos no pertenecen a Salticidae.

Sin lugar a dudas, los registros más antiguos de Salticidae que se tienen actualmente, son por la presencia de los géneros Almolinus Petrunkevitch, 1958; Gorgopsina Petrunkevitch, 1955 y Prolinus Petrunkevitch, 1958; considerados extintos; de la subfamilia Hisponinae, para el ámbar del Báltico (Maddison, 2015; Dunlop et al., 2018), con una edad estimada de $44-49 \mathrm{Ma}$ (Weitschat y Wichard, 2002 citado en Bodner y Maddison, 2012). Por lo tanto, con base en el registro fósil, se ha sugerido que los salticidos aparecen en la Era Cenozoica (Wunderlich, 2004b; Penney, 2010; Penney y Selden, 2011; Riquelme y Hill, 2013). Por otro lado, los análisis filogenéticos realizados por Maddison y Hedin (2003), Maddison et al. (2014) y un estudio elaborado por Bodner y Maddison (2012), quienes utilizan el método de reloj molecular Bayesiano, datan la edad de Salticidae en $47-$ 57 Ma. No obstante, Penney (2010) menciona que no se debería descartar la posibilidad de encontrar a Salticidae en el Mesozoico, en los registros del Cretácico; ya que existe una gran brecha en nuestro conocimiento sobre la presencia de fauna salticida (o la ausencia de ella), en el ámbar de Francia del Eoceno y el ámbar del Cretáceo más joven (53 Ma - 76, $5 \mathrm{Ma}$ ) (Penney, 2008). Podría ser solo cuestión de tiempo y paciencia, a fin de que un paleoaracnólogo descubra la primera araña mesozoica saltadora. Si esto fuera así, como lo menciona Bodner y Maddison (2012), el descubrimiento de arañas saltadoras para esa época, podría alterar la edad raíz de Salticidae.

Ahora, sobre la divergencia de Salticidae, en la investigación realizada por Bodner y Maddison (2012) se menciona que los principales clados de la familia están restringidos a regiones muy particulares en los distintos continentes (como ya se mencionó, y ocurre lo mismo en el caso de los lapsinos [incluyendo a Galianora marcoi sp. nov.], quienes están restringidos a los trópicos del Nuevo Mundo [Maddison, 20015]); sugiriendo así que sus radiaciones probablemente se dan después de la separación continental en el Mesozoico. Consecuentemente, con base en las edades estipuladas para la familia (47 - $57 \mathrm{Ma})$, Bodner y Maddison (2012) mencionan que la diversificación temprana ocurrió en el Eoceno, durante la expansión de los bosques Megatermales y probablemente por el aumento de sus presas. Comenzando esto en el Paleoceno (Paleógeno) (alrededor de 66 $\mathrm{Ma}$ ), cuando ocurre la expansión de los bosques tropicales debido al calentamiento de la Tierra; persistiendo esto a lo largo del Eoceno (Paleógeno) (Morley, 2000 citado en Bodner y Maddison, 2012).

Sobre este tópico, Hill y Richman (2009) también sugirieron que la divergencia y rápida especiación de las arañas saltadoras probablemente ocurrió entre el Paleoceno (Paleógeno) y el Mioceno (Neogéno). La diversidad de las subfamilias en Salticidae y la presencia de formas relativamente modernas de salticidos en el ámbar depositado en América Central, indica una mayor divergencia dentro de los trópicos desde el Mioceno (Riquelme y Hill, 2013). La riqueza de especies de Salticidae encontradas en el ámbar de República Dominicana y las especies reportadas para el ámbar de Chiapas (ambas del Neogeno), incluyendo a Galianora marcoi sp. nov., es consistente con una subsecuente radiación en el Neogeno, no solo con implicaciones Neárticas, como el caso del género Maevia? (Riquelme y Menéndez-Acuña, 2017), sino también con una distribución más al sur en la región Neotropical, como es el caso de Galianora.

\subsection{EVOLUGIÓN GEOLÓGIGA: DISPERSIÓN Y/O PERMANENGIA DE ARANEAE.}

Entonces, se tienen dos géneros en el registro fósil chiapaneco, cuyos representantes actuales están aparentemente restringidos a una región Neártica (Maevia?) y otros más hacia el Sur, en la región Neotropical (Galianora). Indudablemente la historia geológica en América Central es una pieza clave para entender su posible distribución. La evolución tectónica y sedimentaria de lo 
que conocemos como América Central incluye México, Centroamérica y las Antillas (para una mejor comprensión del tema ver James, 2007); definitivamente esta evolución afectó y modificó no solo el ambiente sino también el clima en estas regiones. La evolución geológica de Chiapas, desde el Oligoceno hasta el Plioceno, también está vinculada con la formación de América Central (Meneses-Rocha, 2001; Mandujano-Velázquez y Keppie, 2009 citado en Riquelme y Hill, 2013). Así que, los fenómenos tectónicos y los materiales sedimentarios que formaron las actuales montañas en Chiapas, durante el Oligoceno-Mioceno, alteraron la distribución de las cuencas y modificaron las barreras de dispersión (Meneses-Rocha, 2001 citado en Riquelme y Hill, 2013); con base en esto, Riquelme y Hill (2013) mencionan que esto provocó cambios en los ecosistemas locales y representó un potencial impulsor para la diversificación, forzando la dispersión de la paleobiota en Chiapas (incluyendo a Salticidae). Así que en la actualidad, géneros de salticidos como Maevia? y Galianora, ocupan regiones norteñas y sureñas en el Nuevo Mundo, respectivamente.

Actualmente, elárea donde se encuentran las minas de ámbar en Simojovel presenta un clima cálido subhúmedo, con lluvias en verano y la vegetación es de selva mediana (Inafed, 2017), pero hace 23 Ma era una zona de Manglar, con un clima más tropical (Langenheim, 1995; Serrano-Sánchez et al., 2015 Figura 11). Es importante mencionar que el ámbar de República Dominicana comparte similitudes con el de Chiapas, son aproximadamente contemporáneos y ocurre en escenarios similares (Grimaldi, 1996); sin embargo, el ámbar dominicano fue depositado, probablemente, en un complejo de laguna costera (Iturralde-Vinent y MacPhee, 1996). En estos depósitos ambarinos la araneofauna fósil ha sido ampliamente estudiada (e.g. Ono, 1981; Schawaller, 1984; Reiskind, 1986; Wolff, 1990; Wunderlich, 2004a, 2004b, 2004c, 2004d, 2004e, 2004f, 2004g, 2004h, 2004i, 2004j, 2004k, 2004l; Huber y Wunderlich, 2006; Penney, 1999, 2000, 2001, 2005a, 2005b, 2009; Wunderlich, 2011; Poinar Jr, 2015). Es más,
Wunderlich (2004m), Penney (2008) y Penney y Selden (2011) sugirieron que casi todas las familias de arañas fósiles y sus géneros, aún ocupan la misma zona geográfica; para Riquelme y Hill (2013) este tema es debatible.

Nudds y Selden (2008) compararon la biota fósil en el ámbar dominicano con la actual biota de esa región y sugieren que la paleobiota en el ámbar de República Dominicana fue forzada a dispersarse y extinguirse debido a las fluctuaciones climáticas durante la edad de hielo (Neogeno al Pleistoceno). Siguiendo el mismo principio, Riquelme y Hill (2013) mencionan que al parecer la dispersión y extinción de la paleobiota en ámbar de Chiapas se produjo en alopatria por cambios en la tierra y que eso fue seguido por una dispersión forzada a nuevas áreas durante largos periodos de tiempo que abarcaron del Mioceno al Plioceno.

En el contexto anterior, debemos considerar el estudio realizado por Penney y Pérez-Gelabert (2002) sobre la araneofauna fósil y reciente de la Hispaniola (que incluye a República Dominicana y Haití). En ese trabajo se provee un listado de las especies fósiles y actuales de arañas hispaniolicas; las 29 familias encontradas en ámbar, así como 28 de esos géneros están presentes actualmente en esa región; sin embargo, 25 géneros y todas las especies descritas hasta el momento, aparentemente están extintos. Penney y Pérez-Gelabert (2002) mencionaron que las familias presentes en el ámbar dominicano, pero no reportadas para la actual araneofauna hispaniolica, como Cyrtaucheniidae, Ochyroceratidae, Tetrablemmidae, Hersiliidae, Anapidae y Mysmenidae, pueden predecir que tienen representantes actuales en la Hispaniola.

Pérez-Gelabert (2008), elabora un listado, con base en la literatura publicada hasta 2007, de los artrópodos actuales y fósiles de la Hispaniola (República Dominicana y Haiti), incluyendo arañas, él menciona los siguientes registros para la araneofauna actual de esa región, Yabisi guaba Rheims y Brecovit, 2004 (Hersillidae), Ochyrocera cachote Hormiga et al., 2007 (Ochyroceratidae) y Microdipoens sp. (Mysmenidae, en Hormiga et al., 2007); de esta manera se corrobora lo antes 
mencionado por Penney y Pérez-Gelabert (2002). Gabriel de los Santos (com. pers., 2017) menciona que los registros de arañas para la Hispaniola han aumentado considerablemente después de lo publicado por Pérez-Gelabert (2008), pasando ya las 420 especies; pero esta última cifra todavía resulta desactualizada, ya que hace falta incluir nuevas descripciones y realizar una revisión bibliográfica más minuciosa de lo que ya se ha registrado. Probablemente, los nuevos y futuros hallazgos traigan consigo innovadora información sobre el conocimiento de la araneofauna actual y fósil en esa región, que tendrá implicaciones a nivel mundial.

Algo similar ocurre con la araneofauna fósil en Chiapas; en donde 11 géneros, y por lo tanto las especies que los componen, son considerados extintos (ver Dunlop et al., 2018) y seis de ellos tienen representantes en la fauna actual, tal es el caso de Hemirrhagus (Teraphosidae, en García-Villfuerte, 2008), Lyssomanes (Salticidae, en García-Villafuerte y Penney, 2003), Episinus (Theridiidae, en GarcíaVillafuerte, 2006a) y Selenops (Selenopidae, en García-Villafuerte, 2006b). Así que, siguiendo el pensamiento de Penney y Pérez-Gelabert (2002), no se puede predecir que las familias araneidas (y sus componentes), que se encuentran en la actual araneofauna chiapaneca, vayan a ser descubiertas en el ámbar mexicano proveniente de Simojovel (aunque existe una gran probabilidad, dada la evidencia que se tiene por los hallazgos ya realizados), pero con base en el registro de arañas fósiles y actuales de Chiapas (García-Villafuerte, 2009), existe la posibilidad de que los géneros de las diferentes familias de arañas descubiertos en el ámbar mexicano, aun formen parte de la actual araneofauna en Chiapas.

Por lo tanto, con base en las investigaciones realizadas por Penney y Pérez-Gelabert (2002), Hormiga et al. (2007) y Pérez-Gelabert (2008), no solo se debe considerar la evolución geológica y los factores ambientales ya mencionados, para sugerir que la biota fósil dominicana y chiapaneca, y muy en particular la araneofauna incluida en el ámbar de ambos sitios, se ha extinguido y se vio forzada a una dispersión hacia otras áreas, ya que las arañas (así como sus presas, los insectos), han mostrado un fuerte carácter adaptativo a las condiciones cambiantes (Decae, 1984; Dimitrov et al., 2011), y esto es confirmado por la permanencia de representantes no fósiles en la actual araneofauna de la Hispaniola (Pérez-Gelabert, 2008), los cuales fueron primeramente reportados como registros fósiles (e.g. Hersillidae y Mysmenidae).

\section{Conclusiones}

Probablemente muchas de las especies de arañas en ámbar (sino es que todas) que se han encontrado en Chiapas, se extinguieron; pero existe una gran probabilidad, con base en los descubrimientos hechos, que otras especies, representando los mismos géneros y/o familias, hayan ocupado su lugar; pero para fortalecer esta hipótesis, aceptarla o rechazarla, se propone que se incrementen los estudios araneofaunísticos y se adhiera la información a los trabajos previamente realizados (e.g. Ibarra-Núñez et al., 1997; Medina-Soriano, 2005; Santos, 2005; Ibarra-Núñez et al., 2011), que se han centrado en la riqueza y diversidad de las especies de arañas actuales en Chiapas, así como la consideración de estudios filogenéticos con la inclusión de taxa fósiles en ambas áreas. Entonces, si representantes de los géneros Mysmena, Maevia? y Galianora, por mencionar algunos, son encontradas en Chiapas, implicaría que la evolución geológica y los cambios ambientales, por más drásticos que hayan sido, no perturbaron la permanencia de estos géneros en esta región del mundo y que es probable, con base en el registro fósil, que Chiapas fue, en su momento, un centro de origen para muchas especies de arañas, que en la actualidad ocupan diferentes hábitats. Futuras investigaciones relacionadas con arañas fósiles y actuales, podrían confirmar o rechazar esto. 


\section{Agradecimientos}

Quiero agradecer a mis colegas en el Museo de Paleontología "Eliseo Palacios Aguilera", por el gran esfuerzo y dedicación al mantener vivo el espíritu de la paleontología en Chiapas, que se ve reflejado no solamente en el Estado, sino también a nivel Nacional e Internacional: a Roberto Vidal López, Fátima Martínez Guillen, en particular a Gerardo F. Carbot-Chanona por las importantes sugerencias hechas al manuscrito y por la ayuda con la estratigrafía del área de estudio, en especial a Marco A. Coutiño José, quien ha logrado rescatar piezas importantes de ámbar con inclusiones biológicas, lo cual nos ha permitido conocer más sobre la paleobiota chiapaneca y esto nos ha brindado un mejor conocimiento de la actual fauna en la región, y a Luis Enrique Gómez-Pérez por su dedicación, esfuerzo e importante apoyo en cada proyecto abordado dentro del Museo de Paleontología, el resultado final de las fotografias y el mapa en este estudio, se lo debo a él. A Wayne Maddison por cofirmar la identificación del espécimen como parte del género Galianora. Agradezco a Jorge Alfredo Mérida Rivas, del Equipo Abejas del ECOSOR - Unidad San Cristóbal, por permitirme el acceso al equipo de laboratorio en dicha institución. A Gabriel de los Santos, curador de arácnidos, en el departamento de Investigación y Conservación - Museo Nacional de Historia Natural "Prof. Eugenio de Jesús Marcano" (República Dominicana) - por facilitarme valiosa literatura para el desarrollo de esta investigación. A los dos revisores anónimos por los substanciales y acertados comentarios y sugerencias. Gracias a Jason Dunlop por las correcciones y sugerencias hechas al "Abstract". Esta investigación se lleva a cabo como parte del proyecto "Prospección y Resguardo del Patrimonio Paleontológico de Chiapas", financiado por Gobierno del Estado de Chiapas.

\section{Referencias}

Alisson, L.G., 1967, The Cenozoic stratigraphy of Chiapas, Mexico, with discussions of the classification of the Turritellidae and selected Mexican representatives, California: Berkeley, USA, University of California, $\mathrm{PhD}$ thesis, $225 \mathrm{p}$.

Bickel, D.J., Solórzano-Kraemer, M.M., 2016, The Dolichopodidae (Diptera) of Mexican amber: Boletín de la Sociedad Geológica Mexicana, 68, 11-21.

Blackledge, T.A., Scharff, N., Coddington, J.A., Szüts, T., Wenzel, J.W., Hayashi, Y., Agnarsson, I., 2009, Reconstrunting web evolution and spider diversification in the molecular era: Proceddiings of the National Academy os Sciences, 106, 5229-5234.

Blackwall, J., 1841, The difference in the number of eyes with which spiders are provided proposed as the basis of their distribution into tribes; with descriptions of newly discovered species and the characters of a new family and three new genera of spiders: The Transactions of the Linnean Society of London, 18, 601-670.

Bodner, M.R., Maddison, W.P., 2012, The biogeography and age of salticid spider radiations (Araneae: Salticidae): Molecular Phylogenetics and Evolution, 65, 213-240.

Castelo-Calvillo, J.L., 2000, Diversidad de Salticidae (Arachnida: Araneae) en una localidad de selva baja caducifolia del Sur de Jalisco, México: ENEP-Iztacala, México, Universiad Nacional Autónoma de México, Tesis de licenciatura, $181 \mathrm{p}$.

Clerck, C., 1757, Svenska spindlar, uti sina hufvud-slågter indelte samt under några och sextio särskildte arter beskrefne och med illuminerade figurer uplyste, Stockholmiae, $154 \mathrm{p}$. 
Coddintong, J.A., Levi, H.W., 1991, Systematics and Evolution of Spiders (Araneae): Annual Review of Ecology and Systematics, 22, 565-592.

Decae, A.E., 1984, A theory on the origin of spiders and the primitive function of spider silk: Journal of Arachnology, 12, 21-28.

Deeleman-Reinhold, C.L., Floren, A., 2003, Some remarkable, new or little-known pluridentate salticid spiders from Bornean tree canopy (Araneae: Salticidae): Bulletin of the British Arachnological Society, 12(7), 335-344.

Dimitrov, D., Lopardo, L., Giribet, G., Arnedo, M.A., Álvarez-Padilla, F., Hormiga, G., 2011, Tangled in a sparse spider web: single origin of orb weavers and their spinning work unravelled by denser taxonomic sampling: Proceedings of the Royal Society, 279, 13411350. Doi: 10.1098/rspb.2011.2011.

Dunlop, J.A., Penney, D., Jekel, D., 2018, A summary list of fossil spiders and their relatives, en: World Spider Catalog. Natural History Museum Bern, disponible en $<$ http://wsc.nmbe.ch>, accesado 07 Junio 2018.

Frost, S.H., Langenheim, R.L., 1974, Cenozoic reef biofacies, Tertiary larger Foraminifera and scleractinian corals from Chiapas, Mexico: DeKalb (IL), Northern Illiniois University Press, 388 p.

Galiano, M.E., 1963, Las especies americanas de arañas de la familia Salticidae descritas por Eugène Simon: Redescripciones basadas en los ejemplares típicos: Physis, 23, 273-470.

García-Villafuerte, M.A., 2004, Arañas e Insectos fósiles incluidos en ámbar de Simojovel, Chiapas: Chiapas, México, Universidad de Ciencias y Artes de Chiapas, tesis de licenciatura, $148 \mathrm{p}$.

García-Villafuerte, M.A., 2006a, A new fossil Episinus (Araneae, Theridiidae) from Tertiary
Chiapas amber, Mexico: Revista Ibérica de Aracnología, 13, 120-125.

García-Villafuerte, M.A., 2006b, Selenopidae y

Thomisidae (Arachnida: Araneae) en ámbar de Chiapas, México: Boletín Sociedad Entomológica Aragonesa, 38, 209-212.

García-Villafuerte, M.A., 2007, A new fossil Episinus (Araneae, Theridiidae) from Tertiary Chiapas amber, Mexico: Revista Ibérica de Aracnología, 13, 121-124.

García-Villafuerte, M.A., 2008, Primer registro del género Hemirrhagus (Araneae, Theraphosidae) en ámbar del Terciario, Chiapas, México: Revista Ibérica de Aracnología, 16, 43-47.

García-Villafuerte, M.A., 2009, La araneofauna (Araneae) reciente y fósil de Chiapas, México: Revista Mexicana de Biodiversidad, 80, 633-646.

García-Villafuerte, M.A., Locht, A., 2010, Un nueva especie de Hemirrhagus Simon, 1903 (Theraphosidae: Theraphosinae) de Chiapas, México: Revista Ibérica de Aracnología, 18, 81-86.

García-Villafuerte, M.A., Penney D., 2003, Lyssomanes (Araneae, Salticidae) in Oligocene-Miocene Chiapas amber: Journal of Arachnology, 31, 400-404.

Garcilazo-Cruz, U., Álvarez-Padilla F., 2015, Description of a novel mating plug mechanism in spiders and the description of a new species Maeota setastrobilaris (Araneae, Salticidae): ZooKeys, 509, 1-12.

Grimaldi, D.A., 1996, Amber, Window to the past: University of Michigan, Harry N. Abrams, Publishers, The American Museum of Natural History, 216 p.

Grimaldi, D.A., Engel, M.S., Nascimbene, P.C., 2002, Fossiliferous Cretaceous amber from Myanmar (Burma): its rediscovery, biotic diversity, and paleontological significance: American Museum Novitates, 3361, 1-71. 
Harms, D., Dunlop, J.A., 2008, A revisión of he fossil pirate spiders (Arachnida: Araneae: Mimetidae): Paleontology, 52, 779-802.

Heiss, E., 2016, Calisiopsis azteca n. sp., the first Aradidae from mid Miocene Mexican Amber (Hemiptera: Heteroptera): Boletín de la Sociedad Geológica Mexicana, 68, 7-10.

Hernández-Damián, A.L., Calvillo-Canadell, L., Cevallos-Ferriz, S.R.S., 2016, Flor de una nueva especie de Lunania Hook. (Salicaceae sensu lato- Samydeae) incluida en ámbar del Mioceno de Simojovel de Allende, Chiapas, México: Boletín de la Sociedad Geológica Mexicana, 68, 29-36.

Hill, D.E., Richman, D.B., 2009, The evolution of Jumping spiders (Araneae: Salticidae): a review: Peckhamia, 75, 1-7

Hoffman, A., 1976, Relación bibliográfica preliminar de las arañas de México (Arachnida: Araneae): Universidad Nacional Autónoma de México, Instituto de Biologia, $117 \mathrm{p}$.

Hormiga, G., Alvarez-Padilla, F., Benjamin, S.P., 2007, First records of extant Hispaniolan spiders of the families Mysmenidae, Symphytognathidae, and Ochyroceratidae (Araneae), including a new species of Ochyrocera: American Museum Novitates, $3577,1-21$.

Huber, B.A., Wunderlich, J., 2006, Fossil and extant species of the genus Leptopholcus in the Dominican Republic, with the first cases of egg-parasitism in pholcid spiders (Araneae: Pholcidae): Journal of Natural History, 40, 2341-2360.

Ibarra-Núñez, G., 2013, Diversidad de las arañas (Arachnida: Araneae), en Cruz-Angón, A., Melgarejo, E.D., Camacho-Rico, F., NájeraCordero K.C. (eds.), La biodiversidad en Chiapas: Chiapas, México, Comisión
Nacional para el Conocimiento y Uso de la Biodiversidad, Gobierno del Estado de Chiapas, 191-196.

Ibarra-Núñez, G., García, J.A., Moreno, M.A., 1997, Diferencia en la depredación por arañas tejedoras de redes (Arachnida: Araneae) en cafetales del Soconusco, Chiapas, México; condiferentes sistemas de producción (orgánico y convencional), en Memorias XXXII Congreso Nacional de la Sociedad Mexicana de Entomologíca, Metepec, Puebla, 5-6.

Ibarra-Núñez, G., Maya-Morales, J., ChaméVazquez, D., 2011, Las arañas del bosque mesofilo de montaña de la Reserva de la Biosfera Volcán Tacaná, Chiapas, México: Revista Mexicana de Biodiversidad, 82, 1183-1193.

Inafed, 2017, Enciclopedia de los Municipios y Delegaciones de México: Simojovel, Chiapas, disponible en http://www.inafed.gob. mx/work/enciclopedia/EMM07chiapas/ municipios/07081a.html>, consultado 9 de septiembre de 2017.

Iturralde-Vinent, M.A., MacPhee, R.D.E., 1996, Age and paleogeographical origin of Dominican amber: Science, 273, 1850-1852. James, K.H., 2007, Structural geology: From local elements to regional synthesis, en Bundschuh, J., Alvarado, G. (eds.), Central America: Geology, Resources, and Hazards: London, Taylor and Francis, 277-321.

Jiménez, M.L., 1996, Araneae, en LlorenteBousquets,J., García-Aldrete,A.N., GonzálezSoriano (eds.), Biodiversidad, Taxonomía y Biogeografía de Artrópodos de México: Hacia una síntesis de su conocimiento: Universidad Nacional Autónoma de México, D.F, Instituto de Biología, 83-101. 
Judson, M.L.I., 2016, Pseudoscorpions (Arachnida, Chelonethi) in Mexican amber, with a list os extant species associated with mangrove and Hymenaea treess in Chiapas: Boletín de la Sociedad Geológica Mexicana, 68, 57-79.

Kaddumi, H.F., 2005, Amber of Jordan. The oldest prehistoric insects in fossilized resin: Jordan, Eternal River Museum of Natural History, $224 \mathrm{p}$.

Koch, G.L., Berendt, G.C., 1854, Die im Bernstein befindlichen Crutaceen, Myriapoden, Arachniden und Apteren der Vorwelt, en Berendt G.C. (ed.), Die im Bernstein befindlichen Organischen Reste der Vorweld, Berlin, 1, 1-124.

Langenheim, J.H., 1995, Biology of amber producing tres: focus on case studies of Hymenaea and Agathis, en Anderson, K.B., Krelling, J.C. (eds.), AC.S Symposium series 617: Amber, resinite, and fossil resins: Washintong, American Chemical Society, $1-31$.

Logunov, D.V., Azarkina, G.N., 2008, New species of and records for jumping spiders of the subfamily Spartaeinae (Aranei: Salticidae): Arthropoda Selecta, 16, 97-114.

Macadam, G.R., Ross, A.J., 2016, A New Species of Mayfly, Maccafferium annae sp. nov. (Ephemeroptera: Heptageniidae) from Mexican Amber (Miocene): Boletín de la Sociedad Geológica Mexicana, 68, 1-5.

Maddison, W.P., 2006, New lapsiine jumping spiders from Ecuador (Araneae: Salticidae): Zootaxa, 1255, 17-28.

Maddison W.P., 2015, A phylogenetic classification of jumping spiders (Araneae: Salticidae): Journal of Arachnology, 43, 231-292.

Maddison, W.P., Hedin, M.G., 2003, Jumping spider phylogeny (Araneae: Salticidae): Invertebrate Systematics, 17, 529-549.

Maddison, W.P., Li, D., Bodner, M., Zhang, J., Xu, X., Liu, Q., Liu, F., 2014, The deep phylogeny of jumping spiders (Araneae, Salticidae): ZooKeys, 440, 57-87.
Makhan, D., 2007, Soesiladeepakius aschnae gen. et sp. nov. and Soesiladeepakius amrishi gen. et sp. nov. from Suriname (Araneae: Salticidae): Calodema Supplementary Paper, 60, 1-8.

Mandujano-Velázquez, J.J., Keppie, J.D., 2009, Middle Miocene Chiapas fold and thrust belt of Mexico: a result of collision of the Tehuantepec Transform/Ridge with the Middle America Trench: Special Publications of the Geological society, 327, 55-69.

Medina-Soriano, J.A., 2005, Las arañas errantes del manglar de Chiapas, México: México, D.F., Instituto de Biología, Universidad Nacional Autónoma de México, tesis de maestría, $128 \mathrm{p}$.

Meneses-Rocha, JJ., 2001, Tectonic evolution of the Ixtapagraben, an example of a strike-slip basin in southeastern Mexico: implications for regional petroleum systems, en Bartolini, C., Buffler, R.T., Cantú-Chapa, A. (eds.), The Western Gulf of Mexico Basin: Tectonics, sedimentary basins, and petroleum systems: Tulsa, American Association of Petroleum Geologists Memoir, 75, 183-216.

Metzner, H., 2015, Jumping spiders (Arachnida: Araneae: Salticidae) of the world, disponible en <http://www. jumping-spiders.com>, consultado 28 de agosto de 2017.

Morley, R.J., 2000, Origin and Evolution of Tropical Rain Forests: West Sussex, England, Wiley and Sons Ltd, 378 p.

Néraudeau, D., Perrichot, V., Dejax, J., Masure, E., Nel, A., Philippe, M., Moreau, P., Guillocheau, F., Guyot, T., 2002, A new fossil locality with insects in amber and plants (likely Uppermost Albian): Archingeay (Charente-Maritime, France): Geobios, 35, 233-240.

Nudds, J.R., Selden, P.A., 2008, Fossil Ecosystem of North America. A guide to the sites and their extraordinary biotas: USA, Manson Publishing, $288 \mathrm{p}$.

Ono, H., 1981, First record of a crab spider (Thomisidae) from Dominican amber (amber 
collection Stuttgart: Arachnida, Araneae): Stuttgarter Beitrage zur Naturkunde, 73, $1-13$.

Peckham, G.W., Peckham, E.G., 1901, Spiders of the Phidippus group of the family Attidae: Transactions of the Wisconsin Academy of Sciences, Arts and Letters, 13, 282-358.

Penney, D., 1999, Hypotheses for the Recent Hispaniolan spider fauna based on the Dominican Republic amber spider fauna: Journal of Arachnology, 27, 64-70.

Penney, D., 2000, Miocene spiders in Dominican amber (Oonopidae, Mysmenidae): Palaeontology, 43, 343-357.

Penney, D., 2001, Advances in the taxonomy of spiders in Miocene amber from the Dominican Republic (Arthropoda: Araneae): Palaeontology, 44, 987-1009.

Penney, D., 2005a, First fossil Filistatidae: a new species of Misionella in Miocene amber from the Dominican republic: Journal of Arachnology, 33, 93-100.

Penney, D., 2005b, First Caribbean Floricomus (Araneae: Linyphiidae), a new fossil species in Miocene Dominican Republic amber. A new synonymy fro the extant North American fauna: Geologica Acta, 3, 59-64.

Penney, D., 2007, A new fossil oonopid spider, in lowermost Eocene amber from the Paris Basin, with comments on fossil spider assemblage: African Invertebrates, 48, 71-75.

Penney, D., 2008, Dominican Amber Spiders: A comparative Palaeontological-Neontological Approach to Identification, Faunistics, Ecology and Biogeography: Manchester, Siri Scientific Press, 178 p.

Penney, D., 2009, A new spider family record for Hispaniola - a new species of Plectreurys (Araneae: Plectreuridae) in Miocene Dominican amber: Zootaxa, 2144, 65-68.

Penney, D., 2010, The evolution of jumping spiders (Araneae: Salticidae): the palaeontological evidence: Peckhamia, 81, 1-3.

Penney, D., Pérez-Gelabert, D.E., 2002, Comparison of the Recent and Miocene
Hispaniolan spider faunas: Revista Ibérica de Aracnología, 6, 203-223.

Penney, D., Selden, P.A., 2011, Fossil Spiders:

The Evolutionary History of a Mega-diverse Order: Manchester, Siri Scientific Press, 128 p.

Penney, D., Wheater, G.P., Selden, P.A., 2003, Resistance of spiders to Cretaceous - Tertiary extinction events: Evolution, 57, 2599-2607.

Pérez-Gelabert, D.E., 2008, Arthropods of Hispaniola (Dominican Republic and Haiti): A checklist and bibliography: Zootaxa, 1831, $1-530$.

Perrillat, M., Vega, FJ., Coutiño, M.A., 2010, Miocene mollusks from the Simojovel área in Chiapas, southwestern Mexico: Journal of South America Earth Sciences, 30, 111-119.

Petrunkevitch, A.I., 1955, Arachnida, en Moore, R.G. (ed.), Treatise on invertebrate paleontology: Lawrence, Geological Society of America, 42-162 p.

Petrunkevitch, A.I., 1958, Amber spiders in European collections: Transactions of the Connecticut Academy of Arts and Sciences, $41,97-400$.

Petrunkevitch, A.I., 1963, Chiapas amber spider: Entolomology, University of California Entomology, 31, 1-40.

Petrunkevitch, A.I., 1971, Chiapas amber spider 2: Entomology, University of California, 63, $1-44$.

Pickard-Cambridge, O., 1881, On some new genera and species of Araneidea: Proceedings of the Zoological Society of London, 49, 765-775.

Poinar, Jr.G.O., 2015, Pulchellaranea pedunculata n. gen. n. sp. (Araneae: Araneidae), a new genus of spiders with a review of araneid spiders in Cenozoic Dominican amber: Historical Biology, 27, 103-108.

Poinar, Jr.G., Brown, A., 2002, Hymenaea mexicana sp. nov. (Leguminosae: Caesalpinioideae) from Mexican amber indicates Old World connections: Botanical Journal of the Linnean Society, 139, 125-132. 
Popov, Y.A., 2016, Alumeda solorzanokraemerae sp. nov. From Miocene Mexican amber (Hemiptera: Hereroptera, Reduviidae, Emesinae): Boletín de la Sociedad Geológica Mexicana, 68, 23-27.

Prószyński, J., 2015, Monograph of the Salticidae (Araneae) of the World 1995-2012, disponible en <http://peckhamia.com/ Salticidae/Salticidae.php>, accesado 14 de agosto 2017.

Prószyński, J., 2017, Pragmatic classification of the World's Salticidae (Araneae): Ecologica Montenegrina, 12, 1-133.

Prószyński, J., Deeleman-Reinhold, G.L., 2012, Description of some Salticidae (Aranei) from the Malay archipelago. II. Salticidae of Java and Sumatra, with comments on related species: Arthropoda Selecta, 21, 29-60.

Ramírez, M., 2014, The morphology and phylogeny of dionychan spiders (Araneae: Arameomorphae): Bulletin of the American Museum of Natural History, 390, 1-374.

Reiskind, J., 1986, A new Lyssomanes from the Dominican amber and the possible use of insular fossils in building phylogenies, en Actas $\mathrm{X}$ Congreso Internacional de Aracnología, Jaca: Barcelona, España, Barrientos, J.A. (ed.), 1, 423.

Rheims, C.A., Brescovit, A.D., 2004, Revision and cladistic analysis of the spider family Hersiliidae (Arachnida, Araneae) with emphasis on Neotropical and Nearctic species: Insect Systematics and Evolution, 35, 189-239.

Richman, D.B., Edwards, G.B., Gutler, B., 2005, Salticidae, en Ubick, D., Paquin, P., Cushing, P.E., Roth, V. (eds.), Spiders of North America - an identification manual: USA, American Arachnological Society, 205-206.

Richman D.B., Cutler, B., Hill, D.E., 2011, Salticidae of North America, including Mexico: Peckhamia, 95, 1-88.

Riquelme, F., Hill, D.E., 2013, Insights into amber salticids from the Neogene of Middle America, with the first report of Marpissinae
(Araneae: Salticidae) from the Chiapas amber: Peckhamia, 106, 1-5.

Riquelme, F., Menéndez-Acuña, M., 2017, Miocene spider Maevia eureka nov. sp. (Araneae: Salticidae): PeerJ, 5, 1-15, Doi: https://doi.org/10.7717/peerj.3614.

Riquelme, F., Alvarado-Ortega, J., RamosArias, M., Hernández, M., Le Dez, I., LeeWhithigd, T.A., Ruvalcaba-Sil, J.L., 2014, A fossil stemmiulid millipede (Diplopoda: Stemmiulida) From he miocene amber of Simojovel, Chiapas, Mexico: Historical Biology, 26, 415-427.

Rivas, G., Serrano-Sánchez, L., Fega, FJ., 2016, First record of Procaeculus (Acari: Caeculidae) in Miocene amber from Chiapas, Mexico: Boletín de la Sociedad Geológica Mexicana, 68, 87-92.

Ross, A.J., Coutiño M.A., Nel., A, 2016a, The first records of coenagrionid damselflies (Odonata: Zygoptera: Coenagrionidae: Neoerythromma sp. and Nehalennia sp.) from Mexican Amber (Miocene): Boletín de la Sociedad Geológica Mexicana, 68, 81-86.

Ross, A.J., Mellish, C.J.T., Crighton, B., York, P.V., 2016b, A catalogue of the collections of Mexican amber at the Natural History Museum, London and National Museums Scotland, Edinburgh, UK: Boletín de la Sociedad Geológica Mexicana, 68, 45-55.

Ruiz, G.R.S., 2013, Proposal and phylogenetic relationships of Lapsamita, new genus of lapsiines, and description of a new species (Araneae, Salticidae): PLoS One, 8, 1-5. Doi: $\quad$ https://doi.org/10.1371/journal. pone. 0056188

Santos, G.A., 2005, Arañas tejedoras asociadas a los manglares de las Costa de Chiapas, México: México, D.F, Universidad Nacional Autónoma de México, Instituto de Biología, tesis de maestría, $137 \mathrm{p}$.

Schawaller, W, 1984, The family Selenopidae in Dominican amber (Arachnida: Araneae): Stuttgarter Beiträge zur Naturkunde Serie B (Geologie und Paläontologie), 103, 1-18. 
Scheuring, L., 1914, Die Augen der Arachnoideen

II: Zoologischer Jahrbucher für Anatomie, 37, 369-464.

Serrano-Sánchez, M.L., Hegna, T.A., Schaaf, P., Pérez, L., Centeno-García, E., Vega, F.J., 2015, The aquatic and semiaquatic biota in Miocene amber from the Campo La Granja mine (Chiapas, Mexico): Paleoenvironmental implications: Journal of South American Earth Sciences, 62, 243-256.

Serrano-Sánchez, M.L., Guerao, G., CentenoGarcía, E., Vega, F.J., 2016, Crabs (Brachyura: Grapsoidea: Sesarmidae) as inclusions in Lower Miocene amber from Chiapas, Mexico: Boletín de la Sociedad Geológica Mexicana, 68, 37-43.

Simon, E., 1900, Description d'arachnides nouveaux de la famille des Salticidae(Attidae): Annales de la Société Entomologique de Belgique, 46, 24-56, 363-406.

Solórzano-Kraemer, M.M., 2010, Mexican amber, en Penney, D. (ed.), Biodiversity of Fossils in Amber from the Major World Deposits: Manchester, Siri Scientific Press, 42-56.

Vega, F.J., Nyborg, T., Coutiño, M.A., Solé, J., Hernández-Monzón, O., 2009, Neogene Crustacea from Southeastern Mexico: Bulletin of the Mizunami Fossil Museum, 35, 51-69.

Wanless, F.R., 1982, A revision of the spider genus Cocalodes with a description of a new related genus (Araneae: Salticidae): Bulletin of the British Museum of Natural History, 42, 263-298.

Wanless, F.R., 1984, A review of the spider subfamily Spartaeinae nom. n. (Araneae: Salticidae) with descriptions of six new genera: Bulletin of the British Museum of Natural History, 46, 135-205.

Weitschat, W., Wichard, W., 2002, Atlas of plants and animals in Baltic amber: München, Pfeil, $256 \mathrm{p}$.
Wolff, R.J., 1990, A new species of Thiodina (Araneae: Salticidae) from Dominican amber: Acta Zoologica Fennica, 190, 405-408.

Wood, H., 2008, A revision of the assassin spiders of the Eriauchenius gracilicollis group, a clade of spiders endemic to Madagascar (Araneae: Archaeidae): Zoological Journal of the Linnean Society, 152, 255-296.

World Spider Catalog, 2018, World Spider Catalog, Naural History Museum Bern, disponible en <http://wsc.nmbe.ch, version 18.5>, accesado 19 de agosto de 2017.

Wunderlich, J., 2004a, Fossil jumping spiders (Araneae: Salticidae) in Baltic and Dominican amber, with remarks on Salticidae subfamilies, en Wunderlich, J. (ed.), Beiträge zur Araneologie: Hirschber, Verlag Jörg Wunderlich, 1761-1819.

Wunderlich, J., 2004b, The fossil mygalomorph spiders (Araneae) in Baltic and Dominican amber and about extant members of the family Micromygalidae, en Wunderlich, J. (ed.), Beiträge zur Araneologie: Hirschber, Verlag Jörg Wunderlich, 595-631.

Wunderlich, J., 2004c, Fossil spiders (Araneae) of the superfamily Dysderoidea in Baltic and Dominican amber, with revised family diagnoses, en Wunderlich, J. (ed.), Beiträge zur Araneologie: Hirschber, Verlag Jörg Wunderlich, 633-746.

Wunderlich, J., 2004d, Fossil spiders of the family Uloboridae (Araneae) in Baltic and Dominican amber, en Wunderlich, J. (ed.), Beiträge zur Araneologie: Hirschber, Verlag Jörg Wunderlich, 851-886.

Wunderlich, J., 2004e, The fossil spiders (Araneae) of the families Tetragnathidae and Zygiellidae n. stat. in Baltic and Dominican amber, with notes on higher extant and fossil taxa, en Wunderlich, J. (ed.), Beiträge zur Araneologie: Hirschber, Verlag Jörg Wunderlich, 899-955. 
Wunderlich, J., 2004f, Fossil taxa of the family Araneidae (Araneae) inclusively Nephilinae in Baltic and Dominican amber, with the description of a new extinct subfamily and notes on selected extant taxa, en Wunderlich, J. (ed.), Beiträge zur Araneologie: Hirschber, Verlag Jörg Wunderlich, 956-997.

Wunderlich, J., 2004g, The fossil Theridiosomatidae (Araneae) in Baltic and Dominican amber, en Wunderlich, J. (ed.), Beiträge zur Araneologie: Hirschber, Verlag Jörg Wunderlich, 998-1019.

Wunderlich, J., 2004h, Fossil pirate spiders (Araneae: Araneoidea: Mimetidae s.l.) in Baltic and Dominican amber, with notes on intrafamiliar higher taxa, en Wunderlich, J. (ed.), Beiträge zur Araneologie: Hirschber, VerlagJörg Wunderlich, 1249-1278.

Wunderlich, J., 2004i, The fossil spiders of the family Linyphiidae in Baltic and Dominican amber(Araneae: Linyphiidae), en Wunderlich, J. (ed.), Beiträge zur Araneologie: Hirschber, Verlag Jörg Wunderlich, 1298-1373.
Wunderlich, J., 2004j, Fossil spiders of the family Amaurobiidae (Arachnida: Araneae) in Baltic and Dominican amber, en Wunderlich, J. (ed.), Beiträge zur Araneologie: Hirschber, Verlag Jörg Wunderlich, 1377-1379.

Wunderlich, J., 2004k, Fossil spiders of the family Pisauridae (Araneae) in Baltic and Dominican amber, en Wunderlich, J. (ed.), Beiträge zur Araneologie: Hirschber, Verlag Jörg Wunderlich, 1532-1541.

Wunderlich, J., 2004l, The fossil spiders of the family Liocranidae in Baltic and Dominican amber, en Wunderlich, J. (ed.), Beiträge zur Araneologie: Hirschber, Verlag Jörg Wunderlich, 1623-1635.

Wunderlich, J., 2004m, Fossil spiders in amber and copal: Hirschber, Verlag Joerg Wunderlich, $1908 \mathrm{p}$.

Wunderlich, J., 2011, Some fossil spiders in Dominican amber (Araneae: Hersiliidae, Theridiidae, Gnaphosidae): Beiträge zur Araneologie, 6, 461-471. 